\title{
ERFASSUNG VON FriedhÖFEN AM BEISPIEL DEs JoHANNISFRIEDHOFS IN DRESDEN-TOLKEWITZ
}

\author{
Jörg Kuhn
}

$\mathrm{I}$ m Jahr 2015 wurden von der sächsischen Denkmalpflege Mittel zur Verfügung gestellt, die eine Untersuchung des 1881 eröffneten Evangelischen Johannisfriedhofs in Dresden-Tolkewitz unter dem Aspekt der künstlerischen und sepulkralgeschichtlichen Bedeutung und eine daraus folgende kunsthistorische Bewertung ermöglichten. Meiner Untersuchung war eine Erfassung der als denkmalwert eingeschätzten Grabanlagen des Johannisfriedhofs durch Mitarbeiter des Sächsischen Landesamtes für Denkmalpflege in Dresden vorausgegangen. Die etwa 425 Grabanlagen wurden auf einem Plan des 25ha großen Friedhofs eingetragen. $\mathrm{Zu}$ jeder Grabstätte wurde eine kleine Übersichtsaufnahme gefertigt und diese zusammen mit knappen Angaben zum Objekt (Denkmal-Nummer, Lage und Namen, Datierung) in einer Kartei zusammengeführt. Ziel war der erweiterten Untersuchung war es, Grabanlagen aufzuzeigen, die aufgrund der künstlerischen Eigenart und durch ihre besondere Stellung innerhalb der sepulkralen Kunstgeschichte eine Einstufung des Johannisfriedhofs als nationales Kulturerbe ermöglichten. Konnte zudem eine kulturgeschichtlich relevante Persönlichkeit mit dem Grab in Zusammenhang gebracht werden, ließ sich die Einschätzung argumentativ noch verstärken. Auswahlkriterien waren etwa die Einmaligkeit des künstlerischen Entwurfs, prototypische oder charakteristische künstlerische Gestaltung für eine bestimmte künstlerische Gestaltungsphase und auch die Stellung des Grabkunstwerks im Euvre der beteiligten Künstler.

Zunächst wurden Grunddaten erhoben.
Hierzu gehören: Denkmalpflege-Nummer, Name der Grabstätte, Lagebezeichnung, kurze Eingruppierung (Wandgrab o. ä.) und Beschreibung, Name der Hauptleiche, Lebensdaten, Beruf, Funktion, Erstbestattung, wichtige familiäre Beziehungen, Einheirat, Querverweise auf weitere Grabstätten der Familie, Übernahme durch andere Grabeigner, Künstler (Architekt, Bildhauer, Kunstschmied, ausführende Firmen), Datierung, wichtigste Literatur zum Grabmal.

So ist eine Liste von etwa 100 Grabanlagen entstanden, zu der eine knappe Einschätzung und andere nötige Daten zusammengestellt wurden. Darüber hinaus entstand eine detaillierte Karte, auf der die Objekte verortet wurden. Sie gibt gleichzeitig Hinweise auf die Bedeutung der Gräber wieder.

Es hat sich gezeigt, dass es eine Fülle künstlerisch herausragender Grabdenkmäler gibt, die gartengestalterisch in ein hervorragend überliefertes Gesamtensemble innerhalb einer bevorzugten topographischen Lage an der Elbe eingebettet sind. Bestechend ist die Einmaligkeit des erhaltenen Grabmalbestandes bis in die tiefsten Begräbnisfelder hinein. Auch wenn es hier schmerzliche Verluste zu beklagen gibt: Sensationell ist die große Anzahl erhaltener Grabgitter unterschiedlichster Gestaltung. Darüber hinaus wurde deutlich, dass der kunsthistorische Schwerpunkt auf den Grabanlagen der Reformzeit zwischen 1900 und etwa 1925 liegt. Die bedeutenden Grabmalfirmen in Tolkewitz, deren Geschäftshäuser an der Wehlener Straße zum Teil noch heute erhalten sind, haben zusammen mit namhaften Ar- 


\section{JohANNISFriedhof in Dresden-Tolkewitz}

chitekten und Bildhauern einmalige Kunstwerke geschaffen oder Prototypen entwickelt, die die Grabmalkunst besonders vor dem Ersten Weltkrieg weit über die Grenzen Dresdens hinaus beeinflusst haben.
Die Grabanlagen müssen vor dem Hintergrund der hiesigen Kulturgeschichte betrachtet werden, da sie ein markanter Spiegel der Entwicklung sind: Das Dresden der Jahrhundertwende erlebte nicht nur

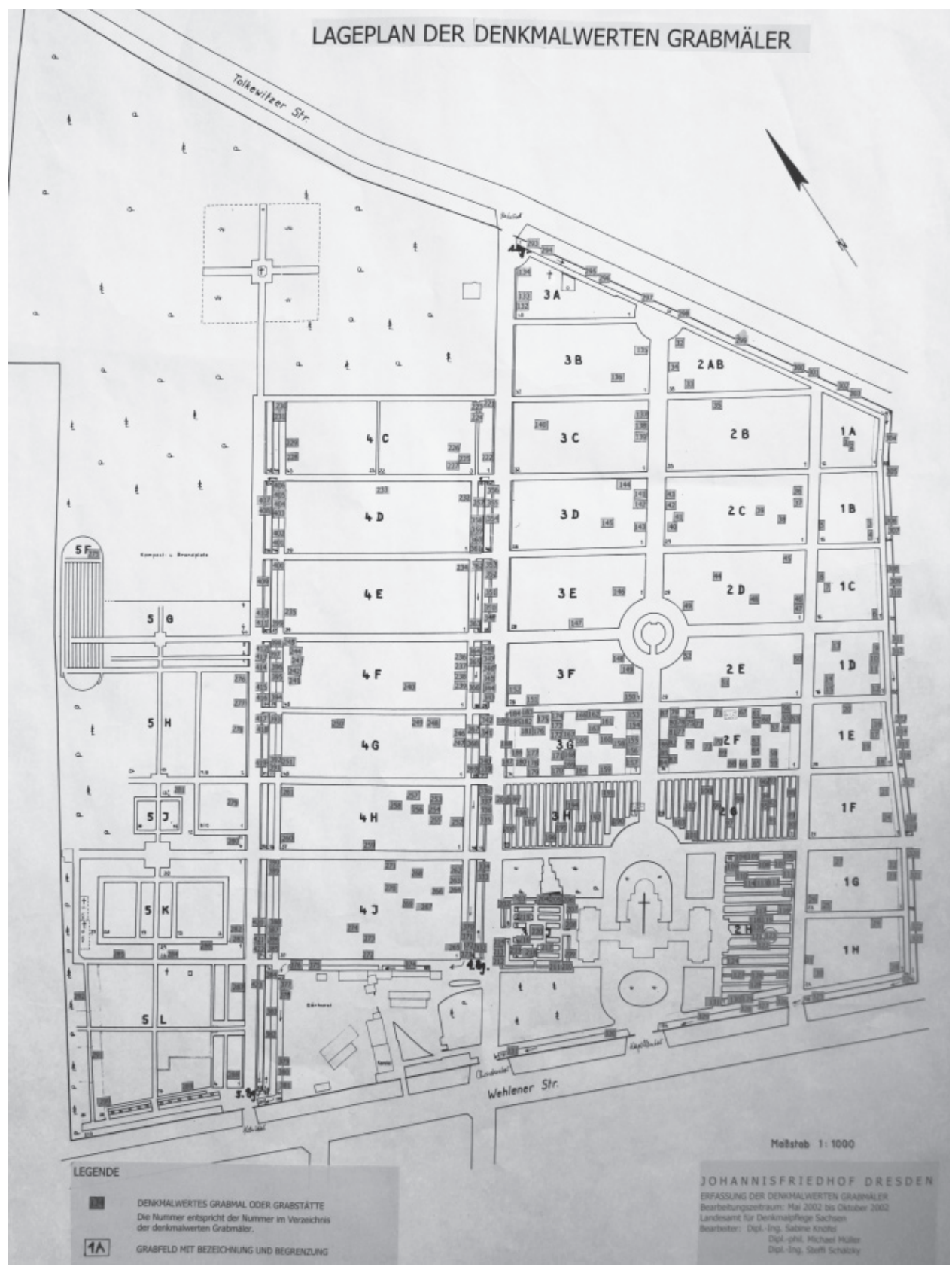

1 | Lageplan des Johannisfriedhofs mit denkmalwerten Grabstätten (Landesamtes für Denkmalpflege Sachsen). 
durch die Industrialisierung einen starken Modernisierungsschub, sondern war auch in künstlerischer Hinsicht eine Keimzelle der Moderne. Eine Reformbewegung in Kunstgewerbe und Architektur nahm hier ihren Ausgang. Ihre wichtigsten Impulse bekam sie von England, vom »Arts and Crafts Movement", das sich gegen die maschinelle Produktion von Kunstgewerbe, Hausrat und Inneneinrichtung wehrte. Bis das reformerische Gedankengut auch im Bereich der Architektur Früchte trug, dauerte es noch eine Weile. Einer der wichtigen Protagonisten war der Architekt und Kunsthistoriker Cornelius Gurlitt. 1902 promovierte Hermann Muthesius als erster Architekt bei ihm an der Technischen Hochschule Dresden. Seit 1901 wirkte an der Hochschule auch der Architekt Fritz Schumacher, der später Hamburgs Stadtbaudirektor werden sollte. Als Architekt sah sich Schumacher, der wie Gurlitt und Muthesius England bereist hatte, als Kulturbringer im Dienst der Gesellschaft. Er war bestrebt, an der Hochschule die noch streng abgegrenzten einzelnen Disziplinen zusammen zu führen. Sein Ziel war das Gesamtkunstwerk, eine Vereinigung aller Künste. Grundlage hierzu bot nach seiner Auffassung die Architektur. Auf ihrem Boden trafen sich Malerei, Bildhauerei und das Kunstgewerbe. Die Reform führte dazu, dass die Dominanz der Architekten im Staatsdienst zugunsten freischaffender Architekten zurückgedrängt wird. Schumacher knüpfte ein Bündnis zwischen Technischer Hochschule und der Kunstgewerbeschule in Dresden. Nicht nur Architekturschüler waren hiervon begeistert. Viele Maler wandten sich unter dem Einfluss der Dresdner Reformbewegung der Architektur zu. 1906 stellte die III. Deutsche Kunstgewerbeausstellung in Dresden - das hier München ablöste - einen Höhepunkt der Reformbewegung dar. Hier beschritt die Dresdner Reformbewegung neue Wege, die die notwendigen Verbindungen zwischen handwerklicher Kunst und industrieller Fertigung schufen. Denn nur so konnten
Viele an der Erneuerung partizipieren. Die Gestaltung von Grabdenkmälern nahm eine besondere Rolle ein. Auch hier wurden neben herausragenden vorbildlichen Einzelwerken die Prototypen für eine gediegene Serienproduktion kleiner, bezahlbarer, aber gestalterisch anspruchsvoller Grabmale entwickelt. Daran beteiligt waren neben Schumacher auch Wilhelm Kreis, sein Bruder Fritz Kreis, Felix Reinhold Voretzsch, Oskar Menzel und andere in Dresden wirkende Architekten, Bildhauer, Kunstschlosser und Maler. Auf dem Johannisfriedhof in Dresden haben sich herausragende Grabgestaltungen dieser reichsweit wirksamen Reformbewegung erhalten.

Unter den 100 als besonders erwähnenswerten Grabstätten konnte ich 23 als besonders herausragende Werke der Grabmalkunst besonders herausstellen (Abb. 1). Vier davon möchte ich im Folgenden exemplarisch vorstellen.

Auch wenn der Schriftsteller Karl May nicht auf dem Johannisfriedhof ruht, gibt es starke Verbindungslinien zwischen May und dem Friedhof. So ruht sein erster Verleger Heinrich Gotthold Münchmeyer ( ${ }^{*} 1836$ Lauterbach bei Bischofswerda, $\uparrow 1892$ Davos) auf dem Johannisfriedhof.

Das außergewöhnliche Jugendstilgrabmal aus Muschelkalkstein ist nach einem Entwurf des Dresdener Baumeisters Paul Colditz entstanden (Abb. 2), möglicherweise eigens zur Aufnahme eines aus Mailand importierten Bronzereliefs des Bildhauers Tullio Brianzi, Schöpfer des Denkmals des einzigen italienischen Nobelpreisträgers Ernesto Teodoro Moneta im Volkspark an der Porta Venezia in Mailand (1924). Das in der Gießerei Teruffini Ottolini Foreto in Mailand gegossene Relief mit dem Seelenführer dürfte das einzige Bronzewerk Brianzis auf einem deutschen Friedhof sein. Es steht hier exemplarisch für die hochrepräsentative Sepulkralkunst Italiens am Ende des 19. und zu Beginn des 20. Jahrhunderts. Die Rahmung des Bildwerks durch die aus Kesseln quellenden Weihrauchschwaden ist originell 


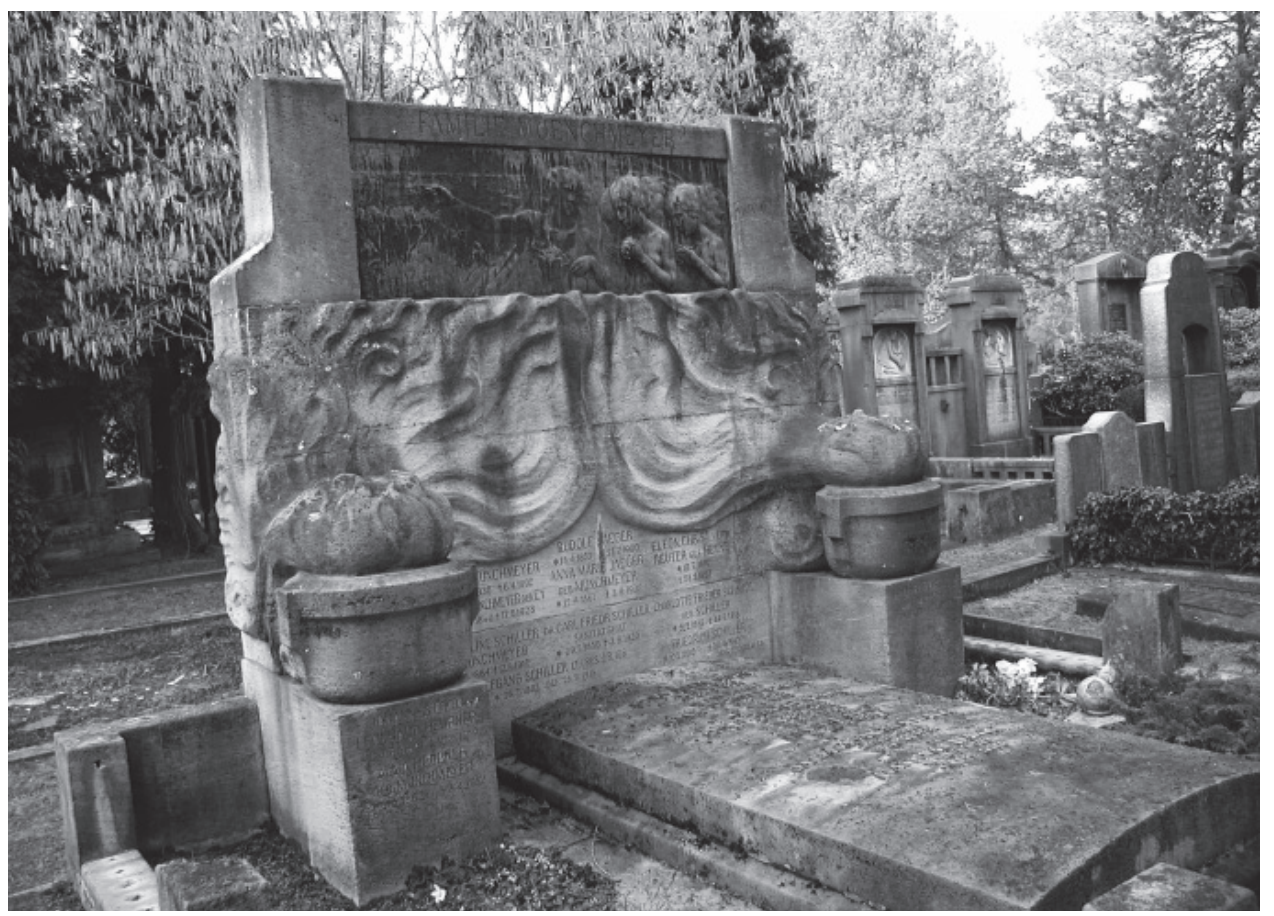

2 | Grabstätte Heinrich Gotthold Münchmeyer, Jäger, Heubner (3.G.06.06), Fotografie (Privat).

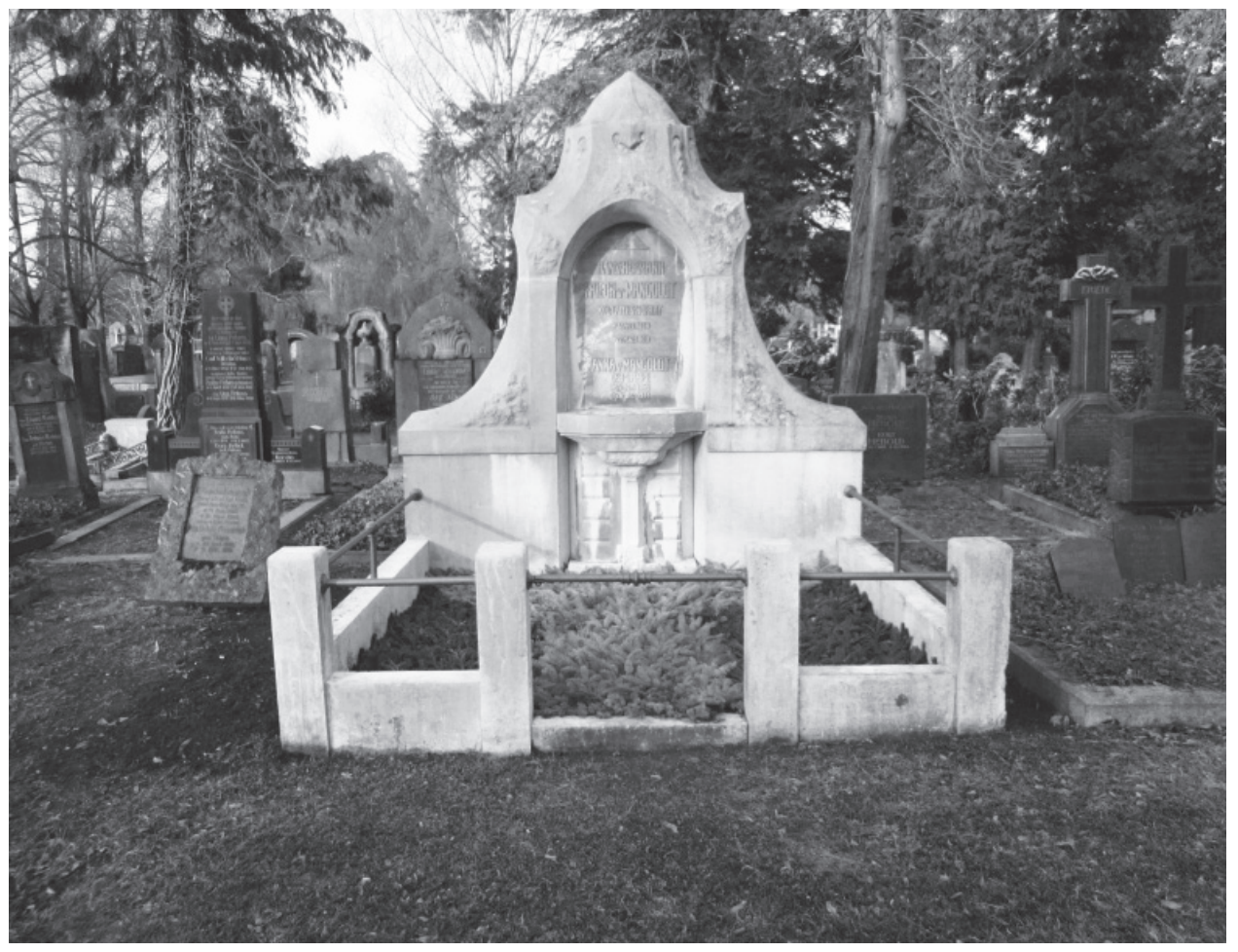

3 | Grabstätte Hofrat von Mangoldt (3.H.01.27-29), Fotografie 2015 (Privat). 
und in ihrer Stilisierung ganz im Sinne eines ornamentalen Jugendstils gestaltet.

Eine andere, noch exklusivere Variante des Jugendstils bringt das Grabmal für den Königlich-Sächsischen Hofarzt Hans Hermann Friedrich von Mangoldt $\left({ }^{\star} 1859\right.$ Göttingen oder Dresden, $\uparrow 1909$ Dresden).

Als der Mediziner und Klinikleiter 1909 starb, beauftragte seine Witwe Anna Amalie von Mangoldt geb. Lampe (1866-1938) den »Erfinder« des deutschen Jugendstils, Hermann Obrist (1862-1927), einen DeutschSchweizer Künstler von internationalem Ruf. Die Verbindung kam - wie nicht selten bei Beauftragungen bei künstlerisch herausragenden Grabdenkmälern - durch familiäre Kontakte zustande. Seit 1898 war Obrist nämlich mit einer Schwester von Frau Mangoldt, Marie-Luise Lampe (1867-1952), verheiratet. Obrist ließ das Grabmal in München durch den Architekten und Steinmetzmeister Alois Aufleger fertigen und nach Dresden transportieren.

Obrist entwickelte wie seine Zeitgenossen Hector Guimard, Louis Majorelle, Henry van de Velde, Antonio Gaudi und Karl Bloßfeldt die Formen seiner Werke aus der Natur. Wobei er stark stilisierte, verfremdete und doch organisch geschlossene Formen erhielt.

Vergleichsbeispiele lassen sich leicht benennen. Ein Brunnen, zuerst in Köln, dann bei Krupp in Essen aufgestellt, verdeutlicht besonders das fantastische, an die skurrilen Architekturen auf den Gemälden des Hieronymus Bosch angelehnte vegetabile Element, das Obrist einbrachte. Ein Grabmal, etwa zeitgleich mit dem Mangoldt-Grab für Carl Ritter von Faber auf dem Münchner Nordfriedhof entstanden, zeigt dagegen stärker die Verarbeitung kristalliner Formen, wie wir es von den Jugendstilkünstlern rund um Max und Bruno Taut, den Brüdern Luckardt und Hans Scharoun her kennen. Der Johannisfriedhof kann sich auf jeden Fall damit brüsten, eines der raren Grabdenkmäler des bekanntesten deutschen Gestalters zu Beginn des 20. Jahrhunderts zu besitzen (Abb. 3).
Für Architekturfans ist das Grabmal Mohr ein Pilgerziel (Abb. 4). Denn der Bauingenieur der Dresdener Technische Hochschule, Geheimrat Otto Mohr (1835-1918), beauftragte nach dem Tod seiner Frau Anna (1851-1907) den wichtigsten Reformarchitekten der Hochschule, Fritz Schumacher. Schumacher. Dieser schuf eine antike Triumpharchitektur, deren Muschelkalkstein-Pfeiler mit Trauerfiguren von Selmar Werner geschmückt werden.

In einer wichtigen zeitgenössischen Architekturzeitschrift, der Deutschen Bauzeitung, wurde 1906 besonders das Grabmal der Familie des Fabrikanten Friedrich Hermann Herzog ( $† 1904)$ als vorbildlich gestaltetes Erbbegräbnis besprochen (Abb. 5). Schöpfer dieser Architektur aus Thüringer Muschelkalkstein war 1905 der junge Dresdner Jugendstilarchitekt Felix Reinhold Voretzsch (1873-1951).

Er zog den wieder neu zu entdeckenden Jugendstilkünstler Ernst Hottenroth (18721908) für die Bauplastik heran und konnte in das Scheinportal der Grabwand eine marmorne Trauernde des Berliner Bildhauers Hans Dammann (1861-1942) stellen. Dammann galt nach 1900 als einer der erfolgreichsten deutschen Grabmalplastiker und der Fachmann für weibliche Trauerfiguren.

Leider ist das von Max Großmann nach Hottenroths Entwurf in Dresden gegossene Torgitter gestohlen worden. Trotzdem kann das Grabmal Herzog noch immer als eine Inkunabel der reformorientierten Jugendstilkunst gelten.

Die wenigen Beispiele müssen hier ausreichen, um einen knappen Eindruck von der herausragenden Bedeutung des Dresdener Johannisfriedhofs zu geben.

Der Johannisfriedhof in Dresden-Tolkewitz ist auf Antrag des Sächsischen Landesamtes für Denkmalschutz auf der Grundlage des vom Verfasser erstellten Gutachtens als Nationales Kulturerbe anerkannt worden. 


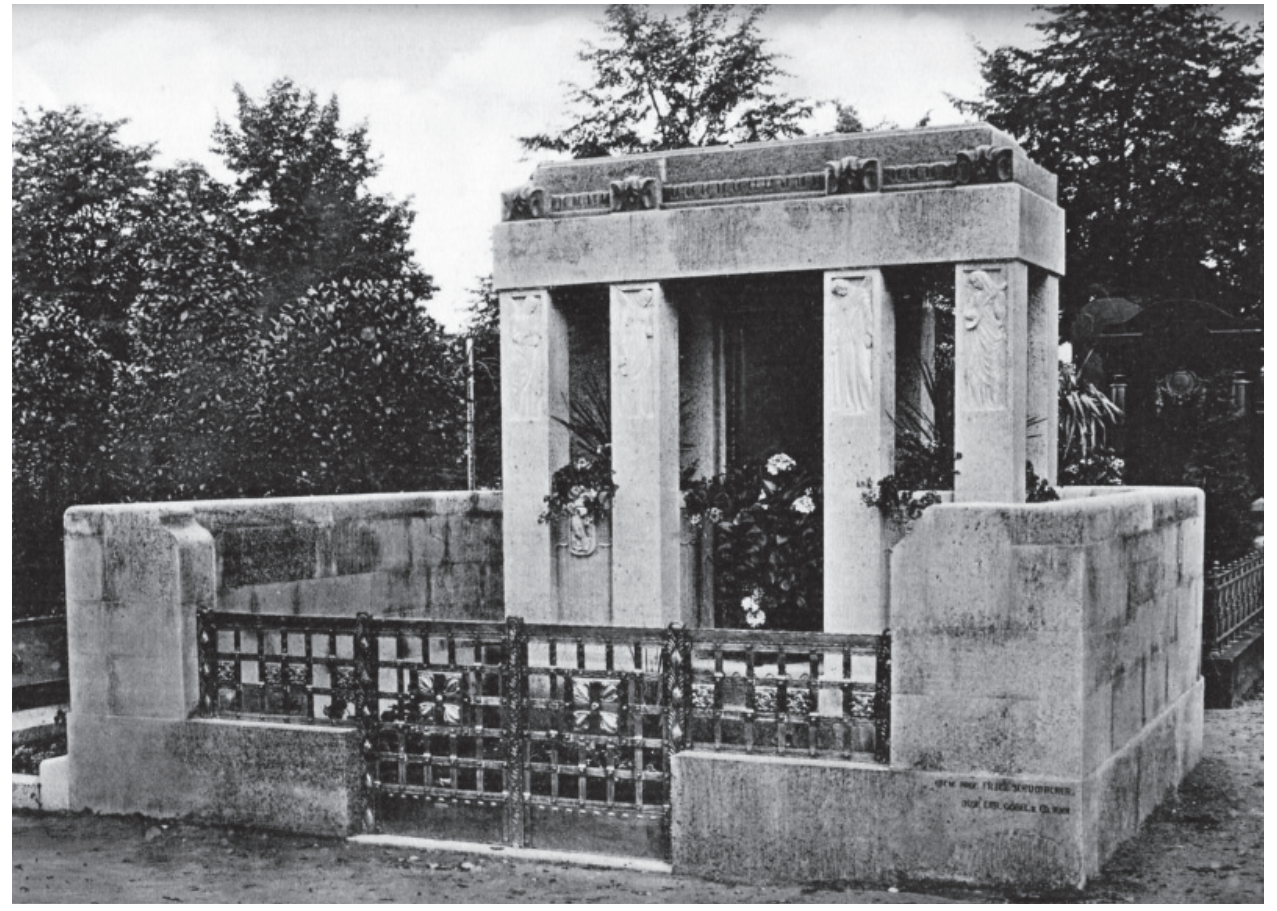

4 | Grabstätte Mohr (2.F.11.12), 1914 (Katalog der Fa. Chr. Göbel \& Co., Dresden).

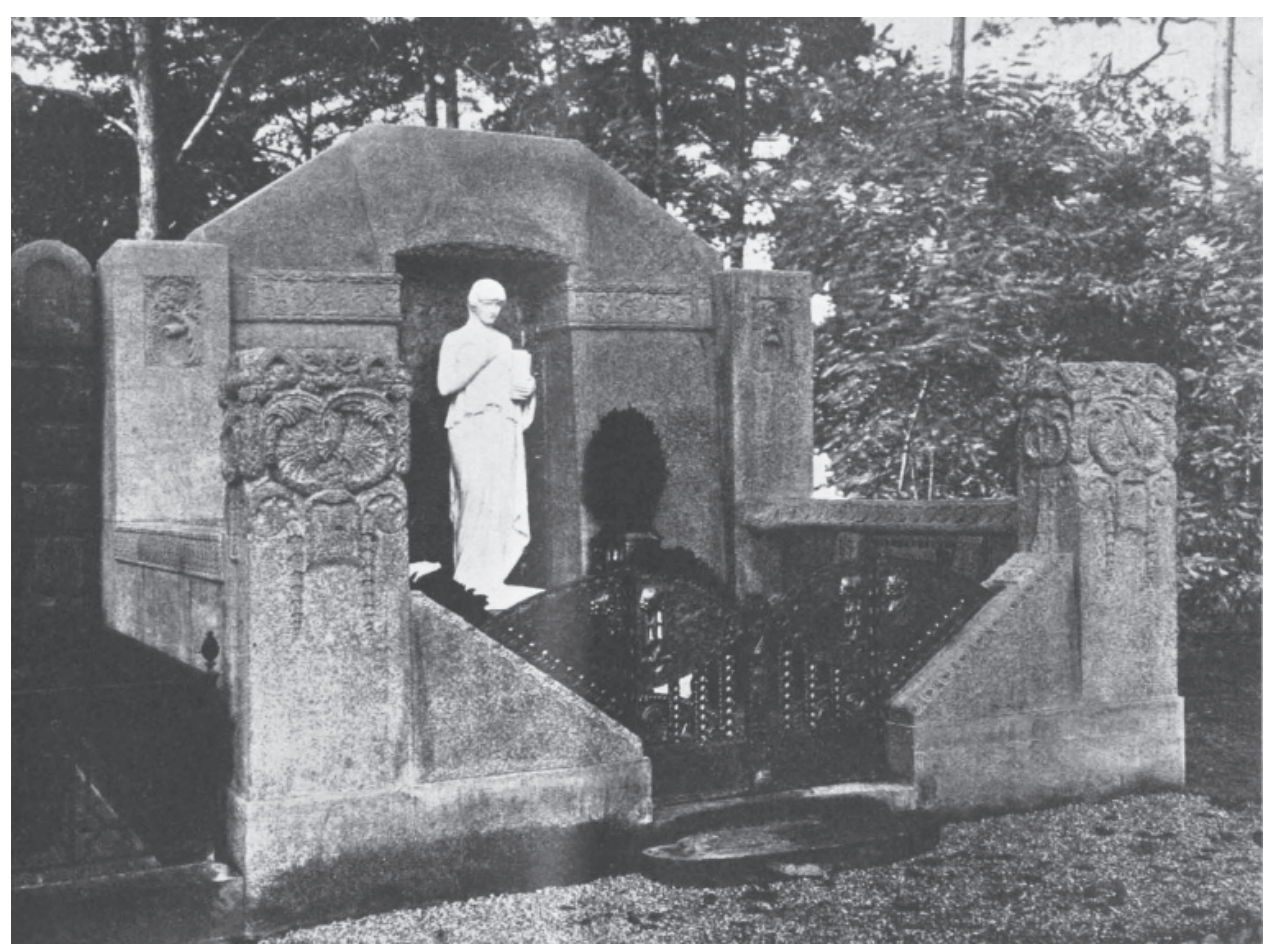

5 | Grabstätte Friedrich Hermann Herzog (4. Bogen 044), Ansicht von 1906 mit Gittertor (Deutsche Bauzeitung, 40. Jg 1906, Nr. 72, S. 642). 